\title{
Implicit solvent model for the interfacial configuration of colloidal nanoparticles and application to the interfacial self-assembly of truncated cubes (SUPPLEMENTARY INFORMATION)
}

\author{
U. Gupta and F. A. Escobedo* \\ School of Chemical and Biomolecular Engineering, Cornell University \\ Ithaca New York 14853
}

\section{QUATERNION-BASED ORIENTATIONAL CONSTRAINT}

In this section, we illustrate one approach for the calculation of the closest angle of approach for the $\{100\}$ feature, $\alpha_{m,\{100\}}$. We start by converting the underlying function [Figure S1 (a)], A=

$\cos ^{-1}\left(\cos \left(\frac{X}{2}\right) \cos \left(\frac{Y}{2}\right)\right)$ to Euler's rotation angle, $\alpha$. This is achieved by transforming the variables, $(\mathrm{X}, \mathrm{Y}) \rightarrow(\theta, \psi)$, according to the Euler angle conventions. We chose the feature orientation, $f=(100)$, given by $\left(\theta_{f}, \psi_{f}\right) \equiv\left(\frac{\pi}{2}, \pi\right)$. Then, for a given NP orientation, $\overrightarrow{\boldsymbol{q}}(\phi, \theta, \psi)$, we generate a set of 24 equivalent (for shapes possessing chiral rotational octahedral symmetry) symmetry-preserving orientations, $\overrightarrow{\boldsymbol{q}}_{i}\left(\phi_{i}, \theta_{i}, \psi_{i}\right)=\overrightarrow{\boldsymbol{q}} \overrightarrow{\boldsymbol{e}}_{\boldsymbol{i}} \forall i \in\{1, \ldots, 24\}$. For each of these we calculate the angle of approach, $\alpha_{f, i}$ given as

$$
\alpha_{f, i}=2 \cos ^{-1}\left(\cos \left(\frac{\theta_{i}-\theta_{f}}{2}\right) \cos \left(\frac{\psi_{i}-\psi_{f}}{2}\right)\right)
$$

$\alpha_{f, i}$ is plotted as a function of $(\theta, \psi)$ in Figure $\mathrm{S} 1(\mathrm{~b})$ as the $i^{\text {th }}$ subplot.

For each $(\theta, \psi)$, a minimum reduction operation is performed across all 24 values of $\alpha_{f, i}$ to calculate the closest angle of approach, $\alpha_{m, f}$. This procedure is repeated to generate the $\left|F_{S}\right|$ basis functions, each corresponding to a feature in the $F_{S}$ set. The three $[\{110\},\{100\},\{111\}]$ used in this work are presented in Figure S1(c) (top to bottom, respectively). The basis functions can then be parameterized (e.g., with a 2parameter harmonic form) and combined (linear sum) in any number of ways to generate the final Potential-of-mean-force (PMF) landscape. 
(a)

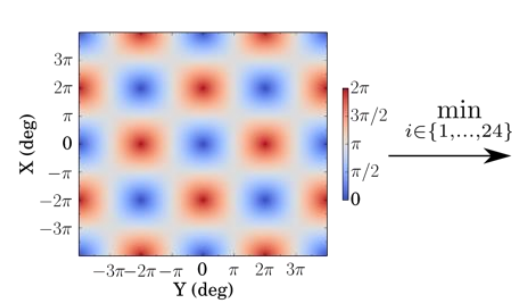

(b)

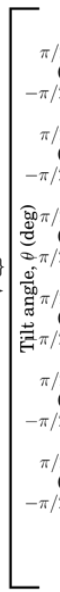

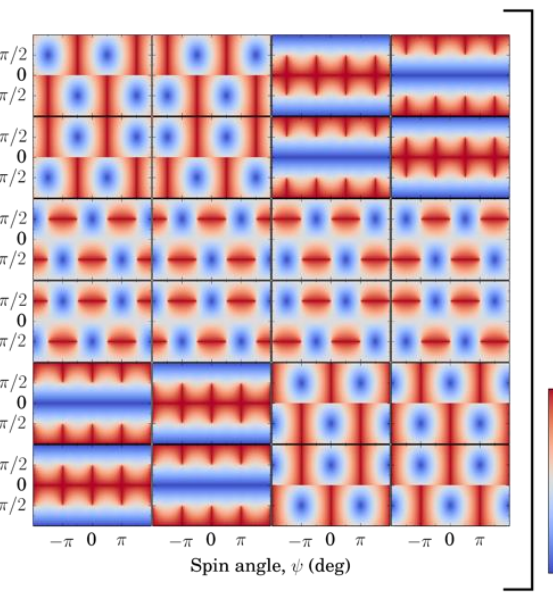

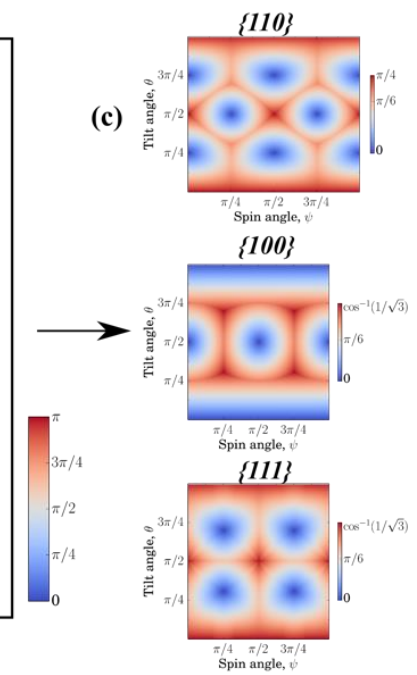

Figure S1: (a) The form of the function, $A=\cos ^{-1}\left(\cos \left(\frac{X}{2}\right) \cos \left(\frac{Y}{2}\right)\right)$, plotted as a function of X and Y. (b) Angle of approach, $\alpha_{\{100\}, i}$ for 24 equivalent orientations (corresponding to chiral rotational octahedral symmetry) as a function of $(\theta, \psi)$. (c) Symmetric reduction of the $24 \alpha_{(100), i}$ functions into the final $\alpha_{m,\{100\}}$ basis function (middle panel). Using the same procedure, the $\alpha_{m,\{110\}}, \alpha_{m,\{111\}}$ basis functions can also be generated (top and bottom panel respectively).

\section{DATA FITTING AND PARAMETER EXTRACTION}

The potential-of-mean-force (PMF) function, $e=E_{t}(S, \overrightarrow{\boldsymbol{q}})$ is fitted to the free energy $(y=F E)$ data using a least-squares regression method. To ensure a good fit in this process, the bias map was first smoothened using a gaussian filter. As a measure of goodness-of-fit, we use the coefficient of determination $\left(R^{2}\right)$ which is given as,

$$
R^{2}=1-\chi^{2}=1-\frac{\sum_{i}\left(y_{i}-e_{i}\right)^{2}}{\sum_{i}\left(y_{i}-\overline{y_{l}}\right)^{2}}
$$

$\chi^{2}$ represents the sum of the square of residuals (numerator) as a proportion of the variance in the FE data (denominator). A value of $R^{2}$ close to one implies that the fitted model perfectly describes the data. Similarly, a value close to, e.g., 0.8 can be interpreted as the model's ability to capture $\sim 80 \%$ of the variance in the data.

For our purposes, a pre-selected feature subset, $X \subseteq\{\{111\},\{110\},\{100\}\}$ is chosen. A combinatorial search is performed across the $\left(2^{3}-1=\right) 7$ subsets (not considering the empty set) to select the best fit with the least number of features. To ensure a good fit near the orientations of interest, we selectively sample the data from basins (circles of $15^{\circ}$ radius in the $\alpha_{f}$ space) centered around the $\{100\},\{111\}$, $\{110\}$ orientations to calculate $R_{S}^{2}$. The $R_{S}^{2}$ statistic is not reported for the RCO shape because the regions 
of interest (FE basins) do not necessarily coincide with the $\{100\},\{111\},\{110\}$ basins. Another metric $R^{2}$ is also calculated by fitting the entirety of the data. It is important to note that, the $R^{2}$ and $R_{S}^{2}$ values are generated from two separate fitting operations, each generating a different set of optimized values for the parameter sets. In Figure S2, we show two examples for the PMF landscape generated using the gaussian form of the potential given by equation $3 \mathrm{~b}$ in the main text. The $R^{2}$ values are consistently higher for this 3-parameter gaussian model than for its 2-parameter harmonic counterpart (see Table 1 in the main text and Figure S3). We also note that the sharp drop in fitness for the geometry close to the RCO occurs because as the FE map becomes "flatter", the simplistic fitting procedure used here becomes less to distinguish between distinct but faded landscape features. A better fit can be achieved by using more advanced regression techniques.

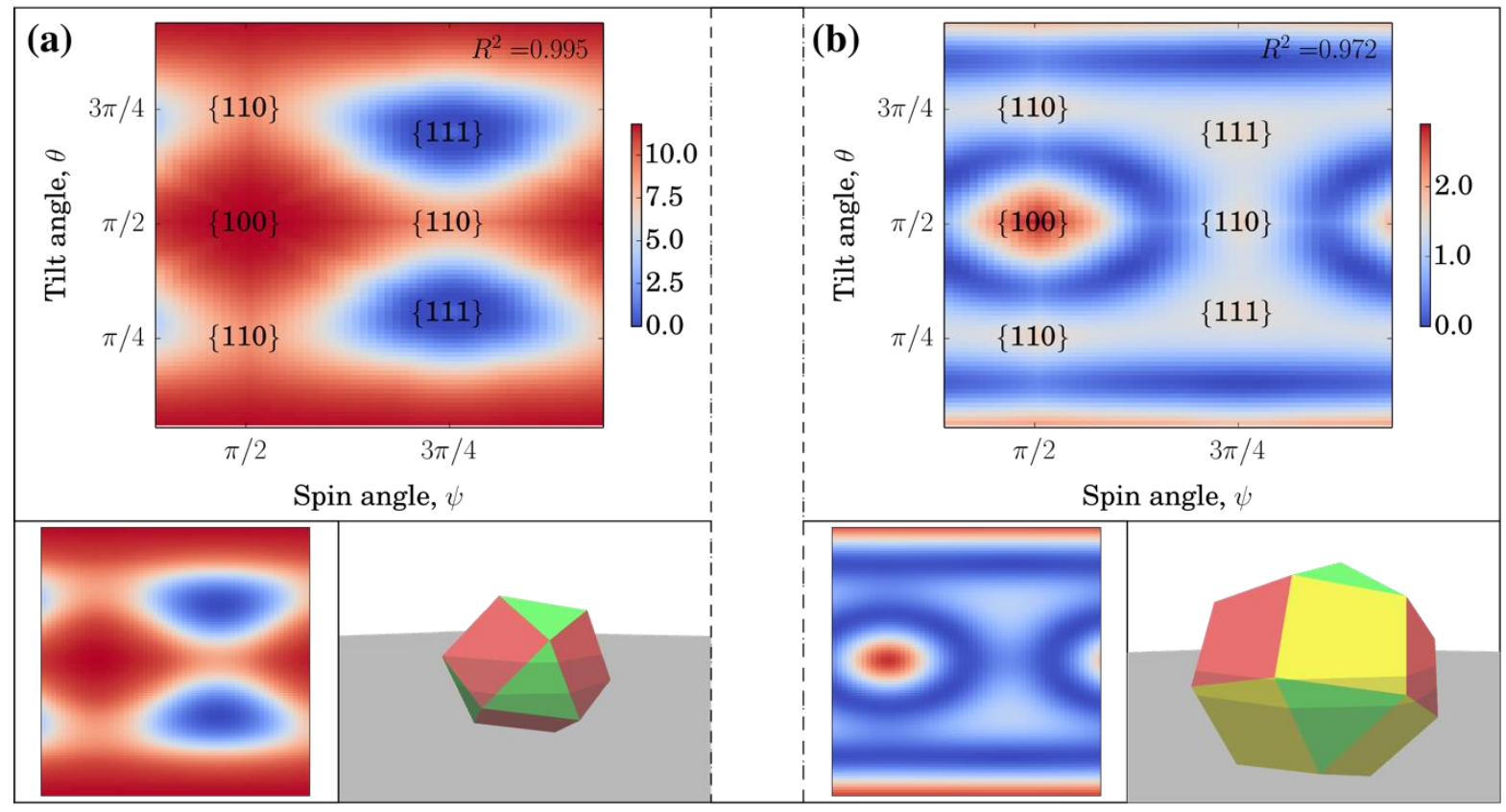

Figure S2: The top panel describes 2-feature gaussian form of the bias potential for (a) CO with $\{111\}$, $\{110\}$ features, and (b) RCO with the $\{100\},\{111\}$ features. The PMF is fitted to the corresponding continuum model results shown in the bottom left panels.

\section{CONTINUUM MODEL AND THE TRUNCATION SERIES}

To display the generalizability of the proposed heuristic PMF model, we consider the fit to the PMF of two series of polyhedra from the family of truncated cubes. A relatively (computationally) inexpensive 
way to estimate the PMF series of truncations is to use a continuum model formulation. We find that a coarser form of model described in in Ref. (1) is sufficient to this end.

The continuum theory describes an isolated NP at the interface of two immiscible fluids where the stacked fluids can be roughly divided into three regions, each with distinct physical properties. The regions (from top to bottom) are the bulk top-solvent (S1), a narrow mixing region (M), and the bulk subphase (S2). The finite central mixing region represents the diffused interface and is responsible for the observed NP preferential configurations. The PMF of the NP in such a theory is given by,

$$
\Delta F(H, \theta, \psi)=\sum_{i \in\{S 1, M, S 2\}}\left(\gamma_{P, i}-\gamma_{P, S 2}\right) A_{P, i}-\left(\beta_{i}-\beta_{S 2}\right) V_{P, i}
$$

where $\gamma_{P, i}$ is the interfacial tension between the NP surface and the $i^{\text {th }}$ fluid phase, and $\beta_{i}$ is the internal energy per unit volume of $i^{\text {th }}$ fluid phase. $A_{P, i}$ and $V_{P, i}$ represent the lateral surface area and the volume of NP in the $i^{\text {th }}$ region respectively. The reference configuration for the system for PMF calculation is defined when the NP completely desorbs from the interface and is present in the bulk of the sub-phase, S2. In this study, we have considered the cases in which the NP interacts equivalently with both the fluids, i.e. $\gamma_{P, S 1}=\gamma_{P, S 2}$ and $\beta_{S 1}=\beta_{S 2}$. Upon substitution, expanding equation S3 leads to,

$$
\Delta F(H, \theta, \psi)=\left(\gamma_{P, M}-\gamma_{P, S 2}\right) A_{P, i}-\left(\beta_{M}-\beta_{S 2}\right) V_{P, i}=\Delta \gamma_{P, M} A_{P, M}-\Delta \beta_{M} V_{P, M}
$$

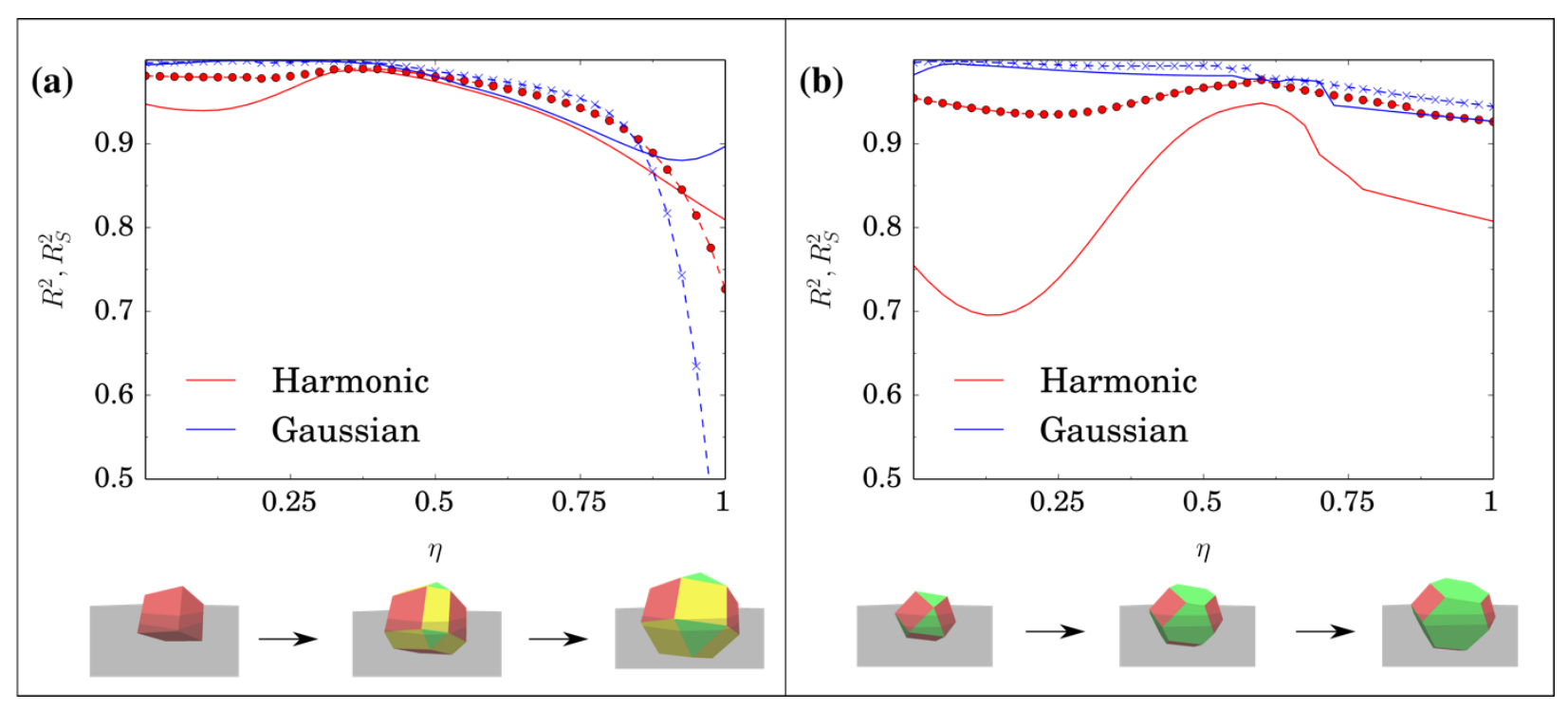

Figure S3: Goodness-of-fit measured in terms of $R^{2}$ (solid line) and $R_{S}^{2}$ (dashed line with symbols) as a function of the truncation, $\eta$. Two forms of the biasing potential, namely harmonic (red) and gaussian (blue), are fitted to the 
PMFs generated using the continuum model. (a) From cube to RCO using the $\{100\},\{110\}$ features, and (b) from CO to TO using the $\{111\},\{110\}$ features.

The parameters $\Delta \beta_{M}$ and $\Delta \gamma_{P, M}$ are extracted from the explicit-solvent Molecular dynamics simulations. ${ }^{1}$ Choosing the values of $\Delta \beta_{M}=3 k_{B} T / \sigma^{3}$ and $\Delta \gamma_{P, M}=-0.5 k_{B} T / \sigma^{2}$ and the width of the interface $W=$ $1 \sigma$, leads to a reasonable fit of the PMF to explicit-solvent MD calculations. Figure S3 shows the coefficient-of-determinations values for the two series of polyhedra fitted using 2-feature orientation biases. In general, we see that the harmonic potential performs reasonably well across the entire series, i.e., with $R^{2}>0.7$. On the other hand, the 3-paramter gaussian potential provides a much better fit $\left(R^{2}>\right.$ $0.9)$ across the board. As expected, the selective fit near regions of interest, $R_{S}^{2}$, is also generally better than $R^{2}$.

\section{MAPPING OF PHYSICAL UNITS}

The reduced units are scaled to physically relevant values by comparing ${ }^{2}$ the generic dimeric fluid used in the explicit-solvent simulations ${ }^{1}$ with a representative solvent like tridecane. Firstly, by equating the density of tridecane ${ }^{3}\left(M=184.361 \frac{\mathrm{g}}{\mathrm{mol}}, \rho=0.7564 \frac{\mathrm{g}}{\mathrm{cm}^{3}}\right.$, therefore $\left.\rho^{*}=\frac{\rho}{M}=4.1 \times 10^{-3} \frac{\mathrm{mol}}{\mathrm{cm}^{3}}\right)$ and that

of a dimeric Lennard-Jones fluid ${ }^{4}\left(\rho^{*}=0.4 \frac{\text { molecules }}{\sigma^{3}}=6.641 \times 10^{-25} \frac{\mathrm{mol}}{\sigma^{3}}\right.$ ), we evaluate the length scale $\sigma$ as $0.55 \mathrm{~nm}$. The mass of a single bead, $m$ is calculated from the density as $1.53 \times 10^{-25} \mathrm{~kg}$. Then, we estimate $\epsilon$ by comparing the critical temperature of tridecane ${ }^{3}\left(T_{c}=676 K\right)$ to that of the dimer ${ }^{4}\left(T_{c}=\right.$ $\left.1.475 \frac{\epsilon}{k_{B}}\right)$, which gives the energy scale $\epsilon$ as $6.44 \times 10^{-21} \mathrm{~J}$. Finally, the time scale, $\tau(=\sigma \sqrt{\mathrm{m} / \epsilon})$ is calculated as $2.7 \mathrm{ps}$.

\section{SIZE SCALING OF THE POLYBEAD MODEL}

For a fixed thickness of the interfacial mixing region (M in Eqs. S3 and S4), the NP size is an important factor in determining its preferred orientation and vertical position about the interface. As is apparent from equation $\mathrm{S} 4$, the size is accounted in the area of contact $\left(A_{P, i}\right)$ and excluded volume $\left(V_{P, i}\right)$ terms. So, as the NP size increases, the corresponding terms increase, leading to deeper FE wells and in certain cases, entirely different (orientation) preferences. ${ }^{1,5}$ We note that the NP size effect is accounted for in the used polybead model as follows. With a change in the number of beads per facet, the excluded volume (from the interfacial region) of the polybead NP scales in perfect accordance with its geometrical/continuum counterpart. Similarly, $\Delta \beta_{M}$ is independent of the NP size as it depends only upon 
the properties of the interface. More importantly, it has been shown ${ }^{1,2}$ that the contact angle, $\theta_{c}$ between an explicit fluid (e.g., a Lennard-Jones dimeric fluid) and a polybead surface (where the surface is composed of discrete CG beads) is independent of the surface size. We ascertain from Young's equation [ $\cos \theta_{c}=\frac{\gamma_{S V}-\gamma_{S L}}{\gamma_{L V}}$, where $\gamma$ is the surface energy of contact between the solid (S), liquid (L) and Vapor (V) phases] that the intrinsic property $\Delta \gamma_{P, M}$ is not dependent on the NP surface size.

Keeping the consideration described above in mind, the implicit solvent parameters $\left(k_{v}, k_{t}\right)$ defining the FE of a larger (or smaller) NP can be generated either by (1) directly simulating the larger NP in an explicit-solvent setting, or (2) fitting a reference explicit-solvent calculation (e.g., for the reported size of $5 \sigma)$ to the continuum model, to generate the size independent parameters $\Delta \beta_{M}$ and $\Delta \gamma_{P, M}$, and using equation S4 to generate the FE map of the larger particle.
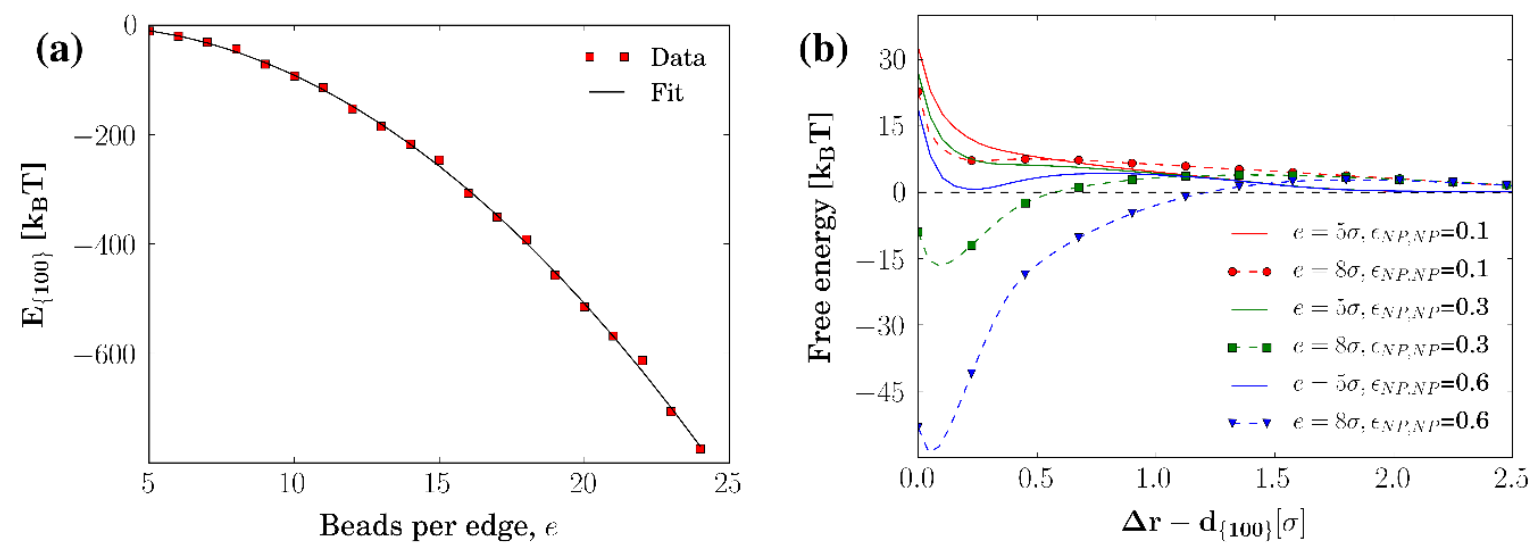

Figure S4: (a) Plot of $E_{\{100\}}$ as a function of NP edge size, e (red square). The scaling is a $2^{\text {nd }}$ order polynomial (black solid line) which represents an effective area of contact between the two $\{100\}$ surfaces. (b) FE is plotted as a function of the distance between NP centers $(\Delta r)$, shifted by the distance of closest approach along the $\{100\}-\{100\}$ contact $\left(d_{\{100\}}\right)$. The solid lines and the dotted lines with the symbols represent the $5 \sigma$ and $8 \sigma$ sizes respectively.

We also note that the polybead model provides reasonable scaling of inter-NP interaction energies with increasing NP size. We define $E_{\{100\}}$ as the total interaction energy between two NPs when their respective $\{100\}$ facets are in complete contact; i.e., when surfaces are placed parallelly at the distance of minimum energy while maintaining maximum contact. This represents the minimum interaction energy possible between the $\{100\}$ facets of the two NPs. Figure S4 (a) shows $E_{\{100\}}$ as a parabolic function of NP edge size. $E_{\{100\}}$ ranges roughly from $10 k_{B} T$ (for $e=5 \sigma$ ) to $100 k_{B} T$ (for $e=10 \sigma$ ) (i.e. $0.5-$ $5 \times 10^{-19} \mathrm{~J}$ based on $\epsilon=6.44 \times 10^{-21} \mathrm{~J}$ ) which is in good agreement with corresponding values for $\{100\}$ facets in PbX NPs. ${ }^{6}$ The $2^{\text {nd }}$ order polynomial, $(e-1)^{2}-3.4(e-1)+3.4$, perfectly accounts for the change of surface energy with edge size, $e$. This expression is easily derived analytically by assuming 
that a single bead in the $\{100\}$ surface of the first NP interacts with only $9(3 \times 3)$ beads of the second NP's aligned $\{100\}$ surface. This is a reasonable assumption considering the length scale and cut-off of the applied Lennard-Jones potential. We also assume that an outer-most (red) bead from the first facet, on average, interacts with a fraction, $f(\in[0,1])$ of the energy of that of a bead close to the center of the facet. Note that our NP model is hollow and is described purely by surface beads. In this context, "outermost" and "close to center" are measures of the radial distance of the beads (that comprise the facet surface) from the facet center. The number of these outer-most beads for a surface width edge, $e$ is given by $(e-1)^{2}-(e-3)^{2}=4(e-2)$. Therefore, if the average energy of bead-bead interaction is given by $\gamma$, the total energy of interaction, $E_{\{100\}}$ can be calculated as,

$$
E_{\{100\}}=\gamma \times(e-3)^{2}+f \gamma \times 4(e-2)=\gamma\left[(e-1)^{2}-4(1-f)(e-1)+4(1-f)\right]
$$

This gives the value of $f$ as $\sim 1 / 6$. For an infinitely large surface, the scaling would have simply been the surface area of contact i.e. $(e-1)^{2}$. However, due to the finite surfaces of the polybead NPs, discontinuity of interactions for the beads at the facet edge cause the scaling to change to an "effective area of contact". We also plot the FE (calculated using Umbrella Sampling ${ }^{7,8}$ ) as a function of inter-NP distance [Figure S4 (b)] for two edge sizes, $e \in\{5 \sigma, 8 \sigma\}$. The CO-shaped NPs are simulated under the conditions predicted by the explicit-solvent calculations [Figure 4 (a)], i.e. $k_{v}=4 \frac{k_{B} T}{\sigma^{2}}$ and $k_{t,\{111\}}=$ $25 \frac{k_{B} T}{\mathrm{rad}^{2}}$. As expected, due to increased surface area of contact, the larger NP has deeper FE wells at short distances of approach as compared to the smaller NP, for the same $\epsilon_{N P, N P}$. However, the shape of the FE curves remains qualitatively the same.

(1) Gupta, U.; Hanrath, T.; Escobedo, F. A. Phys. Rev. Materials 2017, 1 (5), 055602.

(2) Savoy, E. S.; Escobedo, F. A. Langmuir 2012, 28 (7), 3412-3419.

(3) Haynes, W. M. CRC Handbook of Chemistry and Physics, 91 st editi.; Haynes, WM, Ed; 2010.

(4) Cheng, S.; Lechman, J. B.; Plimpton, S. J.; Grest, G. S. J. Chem. Phys. 2011, 134 (22), 224704.

(5) Soligno, G.; Dijkstra, M.; van Roij, R. Phys. Rev. Lett. 2016, 116 (25), 258001-258006.

(6) Zhang, H.; Letters, J. B. T. J. O. P. C.; 2012. ACS Publications 2012, 3 (19), 2882-2886.

(7) Torrie, G. M.; Valleau, J. P. Journal of Computational Physics 1977, 23 (2), 187-199.

(8) Kästner, J. WIREs Comput Mol Sci 2011, 1 (6), 932-942. 